\title{
Antibacterial PMMA Composite Cements with Tunable Thermal and Mechanical Properties
}

Arianna De Mori, ${ }^{\dagger}$ Emanuela Di Gregorio, ${ }^{\dagger}$ Alexander Peter Kao, ${ }^{\dagger}$ Gianluca Tozzi, ${ }^{\ddagger}{ }^{\dagger}$ Eugen Barbu, ${ }^{\dagger}$ Anita Sanghani-Kerai, ${ }^{\S, \|}$ Roger R. Draheim, ${ }^{\dagger}{ }^{\dagger}$ and Marta Roldo*, ${ }^{\dagger}+0$

${ }^{\dagger}$ School of Pharmacy and Biomedical Science, University of Portsmouth, St. Michael’s Building, White Swan Road, PO1 2DT Portsmouth, U.K.

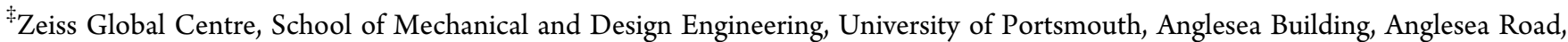
PO1 3DJ Portsmouth, U.K.

${ }^{\S}$ Institute of Orthopaedics and Muscoloskeletal Science, University College of London, Brockley Hill, Stanmore, HA7 4LP London, U.K.

Supporting Information

ABSTRACT: PMMA-based cements are the most used bone cements in vertebroplasty and total hip arthroplasty. However, they present several drawbacks, including susceptibility to bacterial infection, monomer leakage toxicity, and high polymerization temperature, which can all lead to damage to the surrounding tissues and their failure. In the present study, silver nanowires (AgNWs) have been introduced to bestow antibacterial properties; chitosan (CS) to promote porosity and to reduce the polymerization temperature, without negatively affecting the mechanical performance; and methacryloyl chitosan (CSMCC) to promote cross-linking with methyl methacrylate (MMA) and reduce the quantity of monomer required for polymerization. Novel PMMA cements

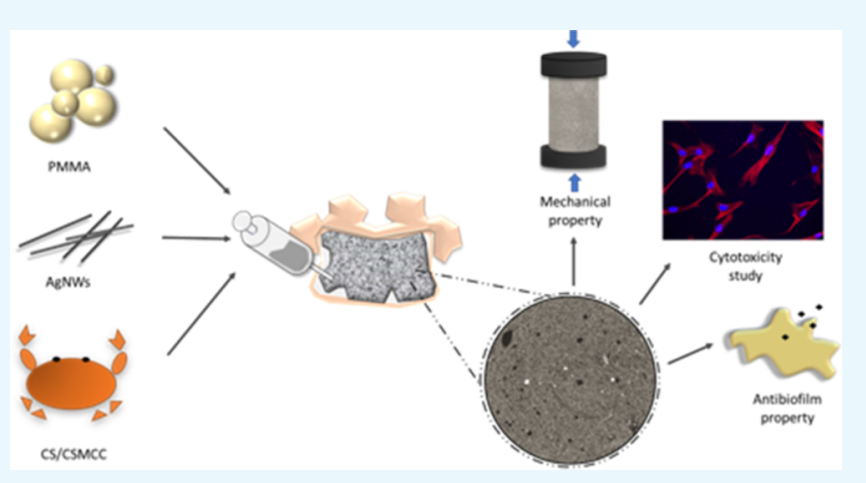
were formulated containing AgNWs (0 and 1\% w/w) and CS or CSMCC at various concentrations $(0,10,20$, and 30\% w/w), testing two different ratios of powder and MMA (P/L). Mechanical, thermal, antibacterial, and cytotoxic properties of the resulting composite cements were tested. Cements with concentrations of CS $>10 \%$ presented a significantly reduced polymerization temperature. The mechanical performances were affected for concentrations $>20 \%$ with a P/L concentration equal to 2:1. Concentrations of AgNWs as low as $1 \% \mathrm{w} / \mathrm{w}$ conferred antimicrobial activity against $S$. aureus, whereas biofilm formation on the surface of the cements was increased when CS was included in the preparation. The combination of CS and AgNWs allowed a higher concentration of $\mathrm{Ag}^{+}$to be released over time with enhanced antimicrobial activity. Inclusion of AgNWs did not affect cell viability on the scaffolds. In conclusion, a combination of CS and AgNWs may be beneficial for reducing both polymerization temperature and biofilm formation, without significantly affecting mesenchymal stem cell proliferation on the scaffolds. No advantages have been noticed as a result of the reducing $\mathrm{P} / \mathrm{L}$ ratio or using CSMCC instead of CS.

\section{INTRODUCTION}

Poly(methyl methacrylate) (PMMA) is currently the most common bone cement used as a screw, prosthesis, or implant fixator in bone, filler for bone cavities or skull defects, and vertebroplasty in osteoporotic patients. ${ }^{1}$ PMMA beads are combined with the liquid monomer (methyl methacrylate, MMA) to form a slurry that, once injected, conforms to the shape of its surrounding, allowing the distribution of implant loads and the formation of strong mechanical bonds with bone following monomer polymerization. Notwithstanding its extensive application, PMMA use is hindered by a number of limitations: lack of bioactivity, poor cement-bone integration, stiffness mismatch between the bone and the cement, high exothermic reaction temperature that can cause tissue necrosis, and monomer toxicity characterized by a sudden drop in blood pressure. ${ }^{2}$ Moreover, it has been reported that MMA can cause allergic reactions through direct contact with the skin (dermatitis) or through inhalation. ${ }^{3}$ Finally, an important risk related to any biomaterial implantation is the occurrence of infections that can lead to biofilm formation and implant failure. $^{4,5}$

One strategy, employed to promote better PMMA bone integration, is to increase the cement porosity with the addition of biodegradable materials. For instance, chitosan can

Received: July 23, 2019

Accepted: October 9, 2019

Published: November 14, 2019 
Table 1. Ratios Employed for the Formulation of Composite Cements, Together with the Average Mass Loss and Water Uptake after 4 Weeks of Incubation in $\mathrm{PBS}^{a}$

\begin{tabular}{|c|c|c|c|c|c|c|c|}
\hline cement type & PMMA (g) & MMA (mL) & CS $(g)$ & $\operatorname{CSMCC}(\mathrm{g})$ & AgNWs (\%) & mass loss (\%) & water uptake (\%) \\
\hline PMMA_1 & 2 & 1 & & & & $0.09 \pm 0.09$ & $2.84 \pm 1.03$ \\
\hline PMMA_0.8 & 2 & 0.8 & & & & $0.12 \pm 0.12$ & $3.44 \pm 0.71$ \\
\hline PMMA_AgNWs_1 & 2 & 1 & & & 1 & $0.23 \pm 0.15$ & $2.76 \pm 0.66$ \\
\hline PMMA_AgNWs_0.8 & 2 & 0.8 & & & 1 & $0.13 \pm 0.22$ & $2.77 \pm 0.73$ \\
\hline PMMA_CS10\%_1 & 1.8 & 1 & 0.2 & & & $0.16 \pm 0.25$ & $3.86 \pm 1.40$ \\
\hline PMMA_CS10\%_0.8 & 1.8 & 0.8 & 0.2 & & & $0.21 \pm 0.24$ & $3.99 \pm 3.93$ \\
\hline PMMA 2:1_CS10\%_AgNWs & 1.8 & 1 & 0.2 & & 1 & $0.11 \pm 0.12$ & $7.15 \pm 4.39^{b}$ \\
\hline PMMA 2:0.8_CS10\%_AgNWs & 1.8 & 0.8 & 0.2 & & 1 & $0.16 \pm 0.01$ & $2.02 \pm 1.31$ \\
\hline PMMA 2:1_CS20\% & 1.6 & 1 & 0.4 & & & $0.62 \pm 1.28$ & $8.70 \pm 2.44^{c}$ \\
\hline PMMA 2:0.8_CS20\% & 1.6 & 0.8 & 0.4 & & & $1.03 \pm 0.65^{b}$ & $9.14 \pm 2.43^{c}$ \\
\hline PMMA 2:1_CS20\%_AgNWs & 1.6 & 1 & 0.4 & & 1 & $1.10 \pm 2.07$ & $10.30 \pm 2.80^{c}$ \\
\hline PMMA 2:0.8_CS20\%_AgNWs & 1.6 & 0.8 & 0.4 & & 1 & $0.93 \pm 0.95^{b}$ & $9.76 \pm 2.10^{c}$ \\
\hline PMMA 2:1_CS30\% & 1.4 & 1 & 0.6 & & & $3.66 \pm 1.03^{c}$ & $13.36 \pm 5.00^{c}$ \\
\hline PMMA 2:0.8_CS30\% & 1.4 & 0.8 & 0.6 & & & $4.00 \pm 1.54^{b}$ & $8.65 \pm 2.96^{c}$ \\
\hline PMMA 2:1_CS30\%_AgNWs & 1.4 & 1 & 0.6 & & 1 & $2.61 \pm 0.25^{d}$ & $11.59 \pm 4.92^{c}$ \\
\hline PMMA 2:0.8_CS30\%_AgNWs & 1.4 & 0.8 & 0.6 & & 1 & $3.29 \pm 0.51^{b}$ & $8.42 \pm 3.71^{b}$ \\
\hline PMMA 2:1_CSMCC10\% & 1.8 & 1 & & 0.2 & & $0.27 \pm 0.27$ & $3.41 \pm 3.09$ \\
\hline PMMA 2:0.8_CSMCC10\% & 1.8 & 0.8 & & 0.2 & & $0.21 \pm 0.30$ & $6.33 \pm 4.29$ \\
\hline PMMA 2:1_CSMCC10\%_AgNWs & 1.8 & 1 & & 0.2 & 1 & $0.21 \pm 0.16$ & $5.49 \pm 1.79$ \\
\hline PMMA 2:0.8_CSMCC10_AgNWs & 1.8 & 0.8 & & 0.2 & 1 & $0.20 \pm 0.07$ & $2.18 \pm 1.57$ \\
\hline PMMA 2:1_CSMCC20\% & 1.6 & 1 & & 0.4 & & $0.32 \pm 1.30$ & $4.85 \pm 0.97$ \\
\hline PMMA 2:0.8_CSMCC20\% & 1.6 & 0.8 & & 0.4 & & $0.42 \pm 0.51$ & $9.22 \pm 2.40^{c}$ \\
\hline PMMA 2:1_CSMCC20\%_AgNWs & 1.6 & 1 & & 0.4 & 1 & $0.53 \pm 0.21$ & $7.60 \pm 1.56^{b}$ \\
\hline PMMA 2:0.8_CSMCC $20 \%$ _AgNWs & 1.6 & 0.8 & & 0.4 & 1 & $1.79 \pm 0.30^{c}$ & $8.57 \pm 2.19$ \\
\hline PMMA 2:1_CSMCC30\% & 1.4 & 1 & & 0.6 & & $2.31 \pm 0.97^{c}$ & $9.09 \pm 3.36^{c}$ \\
\hline PMMA 2:0.8_CSMCC30\% & 1.4 & 0.8 & & 0.6 & & $2.28 \pm 0.73^{b}$ & $8.79 \pm 2.52^{d}$ \\
\hline PMMA 2:1_CSMCC30\%_AgNWs & 1.4 & 1 & & 0.6 & 1 & $3.12 \pm 1.01^{d}$ & $10.68 \pm 1.84^{c}$ \\
\hline PMMA 2:0.8_CSMCC30\%_AgNWs & 1.4 & 0.8 & & 0.6 & 1 & $3.62 \pm 2.57^{b}$ & $9.64 \pm 2.90^{d}$ \\
\hline
\end{tabular}

${ }^{a}$ Weight loss and water uptake are reported as mean $\pm \mathrm{SD}(n \geq 3)$. Dunnett's multiple comparison test was carried out to relate the results from each sample to that of the control (PMMA_2:1). ${ }^{b} p<0.05 .{ }^{c_{p}}<0.01 .{ }^{d} p<0.0001$.

degrade, over time, leaving a rough and porous threedimensional (3D) structure in which the bone can grow, promoting a more stable fixation thanks to the improved interlocking between the bone and cement. ${ }^{6}$ A further advantage of the use of additives in bone cements is their potential to dissipate the heat generated from the polymerization reaction, minimizing the risk of bone necrosis; glycidyl methacrylate (GMA) and trimethoxysilyl propyl methacrylate (3MPMA) have been used to reduce polymerization temperature. ${ }^{7}$ Alternatively, the functionalization of chitosan with glycidyl methacrylate (GMA) to promote cross-linking with MMA can have a similar effect.'

Implantation of PMMA can be complicated by the occurrence of osteomyelitis, a bacterial infection of the bone frequently caused by Staphylococcus aureus. ${ }^{8}$ Infection can lead to the destruction of the bone and consequently to increased rates of treatment failure. ${ }^{9}$ Since more and more bacteria are becoming resistant to antibiotics, ${ }^{10}$ attention has been shifting to alternatives provided by nanotechnology, such as silver nanowires (AgNWs). For example, oleic acid-capped AgNWs $(5.3 \pm 2.3 \mathrm{~nm})$ loaded into PMMA-based cements showed antibacterial activity against methicillin-resistant Staphylococcus aureus (MRSA) and $S$. aureus, at low concentrations of $0.05 \%{ }^{11}$ Slane et al. formulated a PMMA bone cement loaded with silver NPs $(30-50 \mathrm{~nm})$ functionalized with poly(vinylpyrrolidone) at concentrations $0.25,0.5$, and $1 \%$. No antibacterial activity was found in suspension, but all concentrations of AgNPs were able to significantly reduce biofilm growth on the scaffold. ${ }^{12}$ However, all of these systems presented a critical problem related to the extremely poor biodegradability and interconnectivity of PMMA. If the plastic does not degrade, silver will not come in contact with physiological fluids, oxidize, and have an effect on the bacterial cells.

In the present work, we report the preparation and characterization of novel PMMA composites containing chitosan (CS) or methacryloyl chitosan (CSMCC) to reduce polymerization temperature, modify mechanical behavior, and favor the formation of pores, and AgNWs that can offer a prolonged antibacterial effected by a controlled silver ion release, more sustained than spherical nanoparticles. ${ }^{13,14}$ We further investigated the cytocompatibility, antibacterial, and mechanical properties of the composites.

\section{MATERIALS AND METHODS}

Materials. DePuy SmartSet MV medium viscosity bone cement was purchased from eSutures.com (Mokena, IL). Chitosan from shrimp shell low viscosity $\left(M_{\mathrm{n}} 149.9 \pm 4.7 \mathrm{kDa}\right.$, $M_{\mathrm{w}} 170.5 \pm 4.9 \mathrm{kDa}$ as determined by GPC-MALLS, degree of deacetylation $\sim 85 \%$, calculated by ${ }^{1} \mathrm{H}$ NMR), poly(vinylpyrrolidone) powder $(55 \mathrm{kDa})$, and all other reagents, unless otherwise stated, were obtained from Sigma-Aldrich (Irvine, UK). DMEM high glucose with glutaMAX and phenol red, 4',6-diamidino-2-phenylindole dihydrochloride (DAPI), glycerol, heat-inactivated fetal bovine serum, isopropanol, MTT, PBS pH 7.4, phalloidin Dylight 550, trypan blue stain, 
silver nitrate, trypsin $0.25 \%$ EDTA with phenol red, and $\times 100$ penicillin/streptomycin were from Fisher (Loughborough, UK). ATPlite Luminescence ATP detection assay system was purchased from PerkinElmer (Coventry, UK). Deuterium chloride (D, 99.5\%, DCL 20\%), deuterium oxide (D, 99.9\%), and dimethyl sulfoxide- $d_{6}(\mathrm{D}, 99.9 \%)+0.05 \% \mathrm{v} / \mathrm{v}$ tetramethylsilane (TMS) were purchased from Cambridge Isotope Laboratories (Tewksbury, MA).

Synthesis and Characterization of CSMCC. Chitosan (CS, $2 \mathrm{~g}$ ) was homogeneously dispersed in methanesulfonic acid $(11 \mathrm{~mL})$ at $0{ }^{\circ} \mathrm{C}$ with overhead stirring for $2 \mathrm{~h}$. Methacryloyl chloride (MCC, $12 \mathrm{~mL}$ ) was then added dropwise into the chitosan dispersion, and the light brown reaction mixture was further stirred for $4 \mathrm{~h}$ at $0{ }^{\circ} \mathrm{C}$ in the dark. The reaction mixture was poured into cold water, and a light cream precipitate was obtained after neutralization with aqueous ammonia $(35 \% \mathrm{v} / \mathrm{v})$. The precipitate was dialyzed against deionized water for 1 day, and the solid was recovered by centrifugation at $2880 \mathrm{~g}$ for $20 \mathrm{~min}$ at room temperature ( $\times 3)$; the pellet was vacuum-dried at $40{ }^{\circ} \mathrm{C}$ and then pulverized to afford a fine powder.

FT-IR spectra were recorded using a Varian FT-IR 640-IR Instrument (Agilent, Santa Clara, CA), and spectra were processed using Agilent Resolutions Pro software. For ${ }^{1} \mathrm{H}$ NMR analysis, CSMCC was dissolved in DMSO- $d_{6}$ containing $0.5 \%$ TMS standard; chitosan was dissolved in $\mathrm{D}_{2} \mathrm{O}: \mathrm{DCl}$ (98:2); methacryloyl chloride $(200 \mu \mathrm{L})$ was mixed with 800 $\mu \mathrm{L}$ of $\mathrm{CDCl}_{3}$. All of the samples were analyzed using a Jeol Eclipse $+400 \mathrm{MHz}$ NMR instrument (Oxford Instruments, Oxford, UK). The degree of substitution in the reaction product (DS\%) was determined according to the following formula

$$
\operatorname{DS}(\%)=\frac{\left(I_{\mathrm{v} 1}+I_{\mathrm{v} 2}\right)}{2} \times \frac{1}{I_{\mathrm{a}}} \times 100
$$

where $I_{\mathrm{v} 1}$ and $I_{\mathrm{v} 2}$ are the integral intensities of the signals assigned to the two vinylic protons present in methacryloyl chloride, while $I_{\mathrm{a}}$ is the integral intensity of anomeric proton. ${ }^{15}$

The powder particle size was determined by laser diffraction using a Helos particle sizer coupled with a RODOS dry dispersion unit and ASPIROS micro dose module (Sympatec $\mathrm{GmbH}$, Clausthal-Zellerfeld, Germany); a pressure of 2 bar was used for the measurements.

The antibacterial properties of CS and CSMCC against $S$. aureus were assessed in suspension. CS and CSMCC of $10 \mathrm{mg} /$ $\mathrm{mL}$ stock suspensions were prepared in sterile lysogeny broth (LB), further sterilized under UV light overnight, and sonicated for $20 \mathrm{~min}$ at $40 \mathrm{~Hz}$ before testing. Antibacterial studies were carried out according to Ardila et al. with some modifications. ${ }^{16}$ S. aureus (ATCC 25923) $\left(10^{6} \mathrm{CFU} / \mathrm{mL}\right.$ ) was added to CS and CSMCC suspensions (0.2, 0.4, 0.8, 1, 2, 5, and $10 \mathrm{mg} / \mathrm{mL}$ final concentrations), and the volume was brought to $2 \mathrm{~mL}$ with a sterile $\mathrm{LB}$ medium into $8 \mathrm{~mL}$ sterile sealed bottles. After incubation at $37{ }^{\circ} \mathrm{C}$ and shaking at 200 rpm for $12 \mathrm{~h}$ (MaxQ 8000, Thermo Scientific), the bacterial concentration was determined by measuring the optical density of the suspension at $600 \mathrm{~nm}\left(\mathrm{OD}_{600 \mathrm{~nm}}\right)$. Different concentrations of the stock CS and CSMCC suspensions, as well as pure LB, were used as blanks. The experiment was carried out in triplicate $(n=3)$.

Synthesis and Characterization of AgNWs. AgNWs were synthetized using the polyol method, as previously described. ${ }^{14}$ AgNWs, with an average length of $5 \mu \mathrm{m}$ and an average diameter of $99 \mathrm{~nm}$, were obtained. ${ }^{14}$

Preparation of Composite PMMA Cements. Cement formulations with different concentrations of additives and/or powder to liquid monomer ratio (Table 1) have been prepared and tested. Powders, in the selected ratios, were uniformly mixed using a vortex (Fisherbrand) and then mixed with the liquid monomer, at the required $\mathrm{P} / \mathrm{L}$ ratio, by manual mixing with a spatula, until the powders were fully wet. If AgNWs were included in the preparation, freeze-dried AgNWs were suspended in MMA by sonication for at least $20 \mathrm{~min}$ at $40 \mathrm{~Hz}$ before mixing with the powders. When the mixture became dough-like and easier to handle, the whole mixture was transferred into a custom-made 10-well PTFE mold $(6 \mathrm{~mm}$ diameter, $12 \mathrm{~mm}$ height) and manually pressed. The cements were allowed to cure for $1 \mathrm{~h}$ and then removed from the mold and stored in a desiccator at room temperature until further use.

Thermal Behavior. Setting temperature was measured according to Chen et al. with minor adjustments. ${ }^{17} \mathrm{MMA}$ was added to the powders $(3 \mathrm{~g})$ and mixed until the dough was fully wet. This was placed in plastic containers $(3.5 \mathrm{~cm}$ diameter $\times 1 \mathrm{~cm}$ height), and three thermocouples were inserted a few $\mathrm{mm}$ under the surface of the dough to measure and record the temperature at $5 \mathrm{~s}$ intervals for $25 \mathrm{~min}$, using data acquisition software, Pico Log from Pico Technology. Temperature was plotted vs time to determine the peak temperature $\left(T_{\max }\right)$ and setting time $\left(t_{\text {set }}\right)$. The setting time was the time point when the exothermic temperature reached the midpoint temperature between the ambient $\left(T_{\mathrm{amb}}\right)$ and the peak temperatures $\left(T_{\max }\right)$. The setting temperature was determined using the equation below

$$
T_{\mathrm{set}}=\frac{T_{\max }+T_{\mathrm{amb}}}{2}
$$

Degradation Studies. The degradability of the composite cements was evaluated by determining weight loss, water absorbance, and porosity changes following incubation in PBS. Cement specimens were immersed in PBS $(\mathrm{pH}=7.4)$ and placed in an incubator at $37{ }^{\circ} \mathrm{C}$ for 4 weeks, shaking at $90 \mathrm{rpm}$ (Grant, Cambridge, UK); the PBS medium was changed every week. At scheduled time points, samples were removed and weighed (after the removal of any excess liquid). Samples were then dried in a vacuum oven at $37{ }^{\circ} \mathrm{C}$ for 3 days before measuring their final weight. Water absorption and weight loss were determined, applying the equations reported by Kim et al. ${ }^{18}$ The total porosity and pore size were examined using high-resolution X-ray computed tomography, XCT (Xradia 520 Versa, Carl Zeiss X-ray microscopy, CA), and the 3D data was then analyzed with Avizo (9.3.0, FEI Company). The images were collected using a $60 \mathrm{kVp}$ tube voltage, a tube current of $84 \mu \mathrm{A}$, and with a ZEISS LE1 filter in place. With an isotropic voxel size of $6.11 \mu \mathrm{m}, 1601$ projections were collected over $360^{\circ}$ with an exposure time of $3 \mathrm{~s}$ per projection. After reconstruction, a volume of interest was selected and a threshold was applied to segment the pores. From the segmented images, the volume of the pores and the total porosity were calculated. Pores in contact with the edges of the image were excluded from the analysis. Six samples per type were tested for weight loss and water uptake. One sample per type was analyzed three times for porosity and pore size determination. 
A

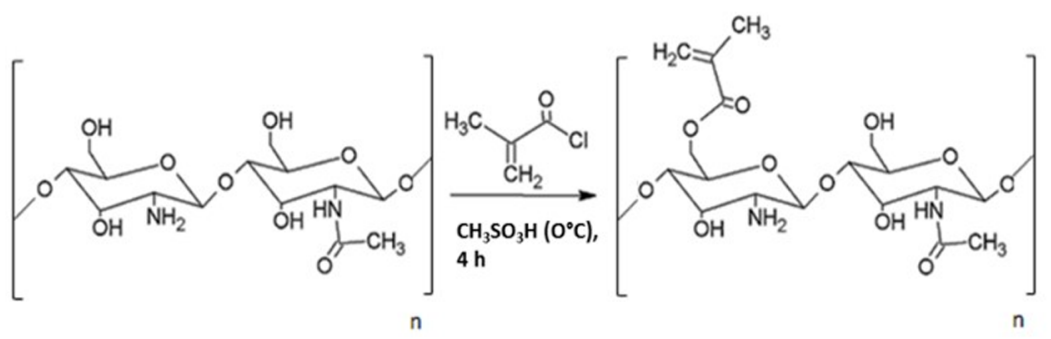

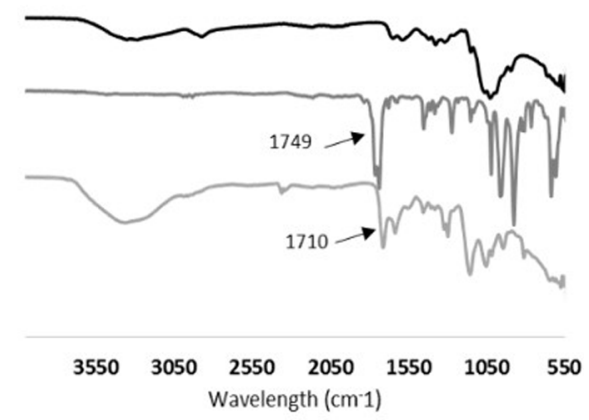

C

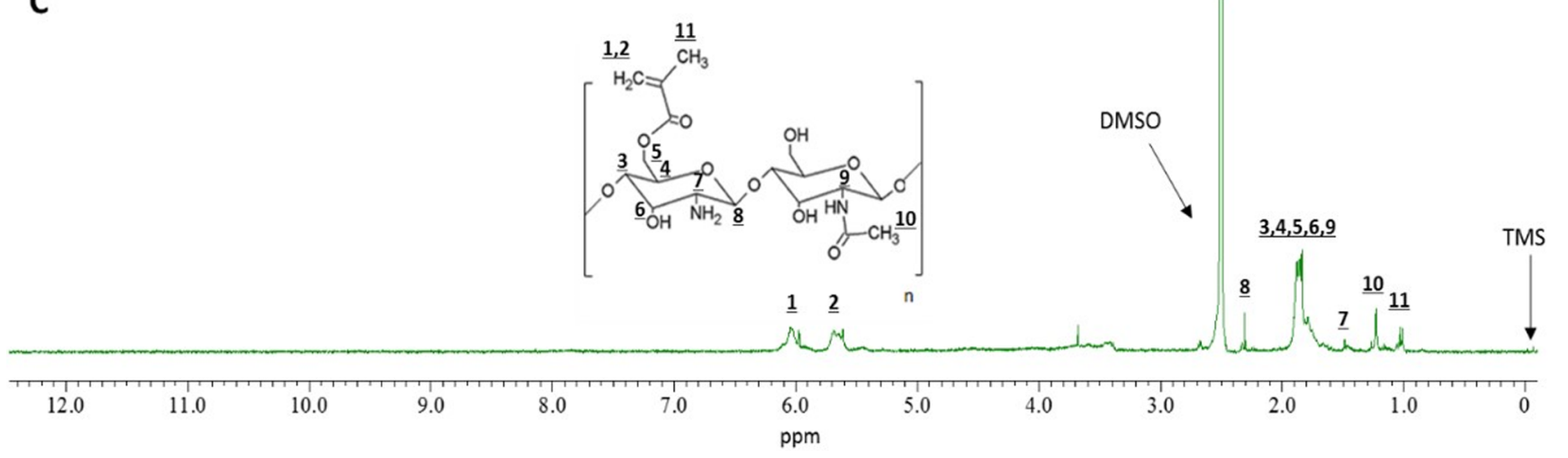

Figure 1. Synthesis and characterization of CSMCC by FT-IR and ${ }^{1} \mathrm{H}$ NMR. (A) Schematic representation of the synthesis of methacryloyl chitosan (CSMCC). (B) FT-IR spectra of CS (black), MCC (dark gray), and CSMCC (light gray). (C) ${ }^{1} \mathrm{H}$ NMR spectrum of CSMCC.

Mechanical Behavior. Compressive tests were carried out to evaluate ultimate compressive strength (UCS) and Young's modulus $(E)$. Cements were prepared in the shape of cylinders as described above, ends were sanded, and the diameter and length were accurately measured using a Vernier caliper. Once cement cylinders were embedded into metallic end caps with epoxy resin using a customized alignment system, they were tested at a speed of $0.02 \mathrm{~mm} / \mathrm{s}$ up to $25 \%$ compression (MTS Bionix, MTS Systems Corp., Eden Prairie, MN). Six samples per cement type were tested, before and after degradation.

Morphological Characterization. The external surfaces of cements before and after degradation were investigated using a high-resolution scanning electron microscope (SEM, Jeol JSM-6160L). Samples were gold-coated using a Polaron e500 instrument (Quoram Technologies, UK). ImageJ (version 1.8.0) software was used to determine the superficial pore size.

Silver Release Studies. The release of silver cations was studied by incubating the cements in $5 \mathrm{~mL}$ of HPLC-grade water at $37{ }^{\circ} \mathrm{C}$ with shaking (90 rpm). At scheduled times, 1 $\mathrm{mL}$ of supernatant was taken and substituted with $1 \mathrm{~mL}$ of freshwater. The concentration of silver ions was determined by a furnace atomic absorption spectrophotometer (VarianSpectrAA 220FS) at a wavelength and spectral bandwidth of 328.1 and $0.2 \mathrm{~nm}$, respectively. A calibration curve was prepared by diluting a silver standard $(1 \mathrm{~g} / \mathrm{L})$ in deionized water (in the range of $1-5 \mathrm{mg} / \mathrm{L}$ ).

Biofilm Formation on Cement Surfaces. Cements were directly prepared in 96-well plates. The day after, they were sterilized by treating with $70 \% \mathrm{EtOH}$ for $30 \mathrm{~min}$ and washing three times in sterile water. Then, they were irradiated with UV light in a laminar flow for $1 \mathrm{~h}$. One hundred microliters of a 1 $\times 10^{6} \mathrm{CFU} / \mathrm{mL}$ suspension of $S$. aureus in the LB medium were plated in each well and incubated for $24 \mathrm{~h}$ at $37^{\circ} \mathrm{C}$. The medium was removed, and each well was gently washed once with PBS to remove loosely adherent bacteria. An MTT assay was carried out to quantify the bacteria adhered to the cement surface: $100 \mu \mathrm{L}$ of $0.5 \mathrm{mg} / \mathrm{mL}$ of the MTT solution in PBS was added to each well, and the plates were incubated for $1 \mathrm{~h}$ in the dark. ${ }^{19}$ The supernatant was removed, and DMSO was added to dissolve the formazan salts inside the cells. Then, the violet solutions were transferred to a new 96-well plate, and the absorbance was read at $570 \mathrm{~nm}$ using DMSO as blank. The experiment was performed in triplicate.

Cytotoxicity Studies. Primary sheep mesenchymal stem cells were obtained under project license number PPL70/8247, and ethical approval was granted by the animal welfare and ethical review board (AWERB) at the Royal Veterinary College, London, UK. Cells were used between passages 2 and 5. Cells were grown in high-glucose DMEM supplemented with $1 \%$ penicillin/streptomycin and $10 \%$ heat-inactivated FBS. Cells were grown until $70 \%$ confluence in an incubator at $37{ }^{\circ} \mathrm{C}$ and $5 \% \mathrm{CO}_{2}$ and then detached with $0.25 \%$ trypsinEDTA. The cell suspension was centrifuged at $400 \mathrm{~g}$ for $5 \mathrm{~min}$ (Eppendorf Centrifuge 5702, UK), and the pellet was resuspended in media, counted, and seeded onto the cylinders. One day after manufacturing, cement cylinders were submerged in complete DMEM at a weight-to-volume ratio of $1: 5$ and incubated at $37^{\circ} \mathrm{C}$ for $24 \mathrm{~h}^{20}$ DMEM was removed and filtered through a $0.22 \mu \mathrm{m}$ filter and frozen until further use; these samples were used to test the biocompatibility of cement extracts. Cells were grown overnight at a cell density of $5 \times 10^{3}$ cells/well in a 96-well plate. Then, the medium was removed, and $100 \mu \mathrm{L}$ of cement extracts were added to each well followed by incubation for 24 and $48 \mathrm{~h}$. At these scheduled time points, the medium was removed and an MTT assay was performed. The cells were plated for $4 \mathrm{~h}$ with 0.5 $\mathrm{mg} / \mathrm{mL}$ MTT in a complete medium. Then, the medium was 
removed, and $100 \mu \mathrm{L} /$ well of DMSO was added. Absorbance was read at $570 \mathrm{~nm}$ using DMSO as blank. The experiment was performed in triplicate.

Cell proliferation on the surface of cements was determined using an ATP bioluminescence assay. Cement cyliders were prepared as described above. Cells (5000 cells/well) were seeded, and their proliferation was measured with an ATP assay, according to the manufacturer's instructions (ATPlite, PerkinElmer). Luminescence readings were taken in a 96-well plate (SpectraMax i3x, Molecular DEVICES). Six replicates per type were tested.

Morphological studies on cells were carried out by a fluorescence microscope after their culture on the cement disks for $24 \mathrm{~h}$. Cells were fixed with paraformaldehyde $4 \%$ in PBS for $15 \mathrm{~min}$, washed with PBS, permeabilized for $10 \mathrm{~min}$ in $0.1 \%$ Triton X-100/PBS, washed twice with PBS, blocked with $2 \%$ bovine serum albumin (BSA) in PBS for $1 \mathrm{~h}$, washed twice with PBS, stained with Phalloidin Dylight 550 in PBS (2 units/ $\mathrm{mL}$, stock solution of 300 units $/ \mathrm{mL}$ in methanol) for $1 \mathrm{~h}(300$ $\mu \mathrm{L}$, at room temperature), washed twice with PBS, stained with DAPI $2 \mu \mathrm{g} / \mathrm{mL}$ in PBS for $10 \mathrm{~min}$, and finally rinsed again with PBS. Photographs were taken, using an epifluorescence microscope (Zeiss Axio Imager $\mathrm{Z} 1$ ) equipped with a Hamamatsu HR camera and a color AxioCam MRc camera. Images were processed by Volocity 6.3 software.

Statistical Analysis. Statistical analyses were performed using GraphPad Prism 7.03 software. Details of the different statistical tests used are reported in the figure captions.

\section{RESULTS}

Physicochemical Characterization of CSMCC. CSMCC was synthesized by modifying the primary hydroxyl group in chitosan with a methacryloyl moiety, via a nucleophilic substitution reaction carried out in a strongly acidic environment (methanesulfonic acid; $\mathrm{p} K_{\mathrm{a}}=-1.9$ ) (Figure 1A). The unreacted methacryloyl chloride was removed during the washing steps as methacrylic acid ammonium salt. ${ }^{21}$ The formation of CSMCC was confirmed by FT-IR (Figure 1B): the new peak at $1710 \mathrm{~cm}^{-1}$ is attributed to the $\mathrm{C}=\mathrm{O}$ bond in the newly formed ester group. The chemical structure of CSMCC was also confirmed by ${ }^{1} \mathrm{H}$ NMR (Figure 1C), with vinylic proton signals appearing at $\delta 5.71$ and $6.02 \mathrm{ppm}$, and methyl protons corresponding to the methacryloyl group appearing at $\delta 1.01 \mathrm{ppm}$. The ${ }^{1} \mathrm{H}$ NMR spectrum also showed a signal at $\delta 1.48 \mathrm{ppm}$ from the three methyl $\mathrm{H}$ atoms $(\mathrm{N}$ acetyl glucosamine), a signal at $\delta 2.24 \mathrm{ppm}$ from $\mathrm{H} 2$ (glucosamine), several overlapping signals (from $\delta 1.7$ to 2.0 ppm) assigned to $\mathrm{H} 3-\mathrm{H} 6$ connected to the non-anomeric C3-C6 carbons in the glucopyranose ring, and $\delta 2.3 \mathrm{ppm}$ from the anomeric proton. The degree of substitution of CSMCC was determined as $25.7 \%$.

In the formulation of composite bone cements, the particle size and shape of the constituent powders are important to guarantee homogeneous mixing and dispersion in the liquid monomer. CS and CSMCC particles presented an irregular shape (Figure S1) and had a significantly higher particle size than the PMMA powder $(p<0.05$, Table 2$)$ that was in the range typically reported for commercial bone cements. ${ }^{22}$

Antibacterial Properties of CS and CSMCC Powders. The bacteriostatic potential of CS and CSMCC powders was tested against $S$. aureus by measuring the absorbance of the bacterial suspension after $12 \mathrm{~h}$ of incubation in LB (Figure 2). The compounds were studied in suspension rather than
Table 2. Average Particle Size, As Determined by Laser Diffraction, Expressed as Sauter Mean Diameter (SMD) and Volume Median Diameter (VMD) of CS, CSMCC, and PMMA Powders ${ }^{a}$

$\begin{array}{llc}\text { material } & \text { SMD }(\mu \mathrm{m}) & \text { VMD }(\mu \mathrm{m}) \\ \text { PMMA } & 34.6 \pm 19.6 & 290.5 \pm 171.6 \\ \text { CS } & 120.3 \pm 21.8^{b} & 533.0 \pm 32.1^{b} \\ \text { CSMCC } & 138.7 \pm 53.9^{b} & 630.3 \pm 39.9^{b c}\end{array}$

${ }^{a}$ The values are reported as mean $\pm \mathrm{SD}(n=3)$. Dunnett's multicomparison test was used to compare SMD and VMD of CS and CSMCC with the ones of PMMA: ${ }^{b} p<0.05$. $t$-test was used to compare CS and CSMCC: ${ }^{c} p<0.05$.

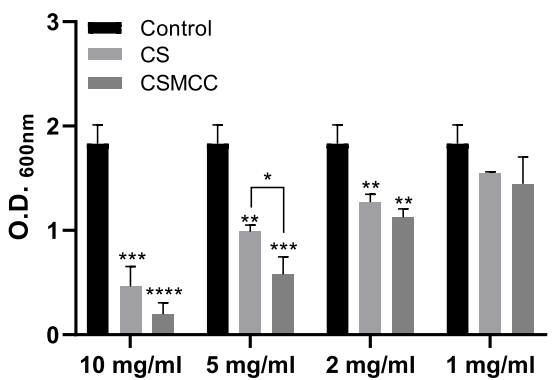

Figure 2. Antibacterial properties of CS and CSMCC. Optical density $\left(\mathrm{OD}_{600 \mathrm{~nm}}\right)$ of $S$. aureus suspensions in the presence of medium as control (black bars), CS (light gray bars), and CSMCC (dark gray bars) in suspension at different concentrations. Data are reported as a mean $\pm \mathrm{SD}(n=3)$. One-way ANOVA returned $p<0.05$; results of the Dunnett's multiple comparison test are reported in the graph $\left({ }^{*} p\right.$ $<0.05 ; * * p<0.01 ; * * * p<0.001 ; * * * * p<0.0001)$. The $t$-test performed between CS and CSMCC at different concentrations revealed no statistical difference $(p>0.05)$.

solution as chitosan and modified chitosan were incorporated in the PMMA cements as solids and are unlikely to dissolve at physiological $\mathrm{pH}$. While bacterial growth was detected for all concentrations studied, results showed that concentrations $\geq 2$ $\mathrm{mg} / \mathrm{mL}$ were required to significantly affect bacterial growth. In this respect, no differences were noticed between the chitosan and modified chitosan.

Experimental Peak Temperature and Setting Time of Cements. Commercially available acrylic bone cement is typically activated by mixing a powder (P) and a liquid component $(\mathrm{L})$ at a $\mathrm{P} / \mathrm{L}$ ratio of $2: 1$. The powder is generally composed of PMMA beads, containing a radical initiator (e.g., benzoyl peroxide) and, when required, a radiopaque agent (e.g., barium sulfate). The liquid contains the monomer methyl methacrylate (MMA), a stabilizer (e.g., hydroquinone), and an accelerator (e.g., $N, N$-dimethyl- $p$-toluidine) to encourage the polymerization reaction to occur at room temperature (cold curing cement).

When the two components are mixed together, the liquid monomer polymerizes around the prepolymerized PMMA particles (Figure 3) generating heat. ${ }^{23}$ In this study, we recorded the polymerization temperature of the composite bone cements and found that the peak polymerization temperature decreased for all composites in comparison with that of the PMMA 2:1 control $\left(66.7 \pm 8.7^{\circ} \mathrm{C}\right.$ ) (Figure 4A,B).

Higher concentrations of CS and CSMCC $(\geq 20 \%$, in particular) induced a significant reduction in the polymerization temperature (CS20\%_0.8, $p<0.01$; CSMCC20\%_1 $p$ $<0.05)$. On the other hand, when the $\mathrm{P} / \mathrm{L}$ ratio decreased to 

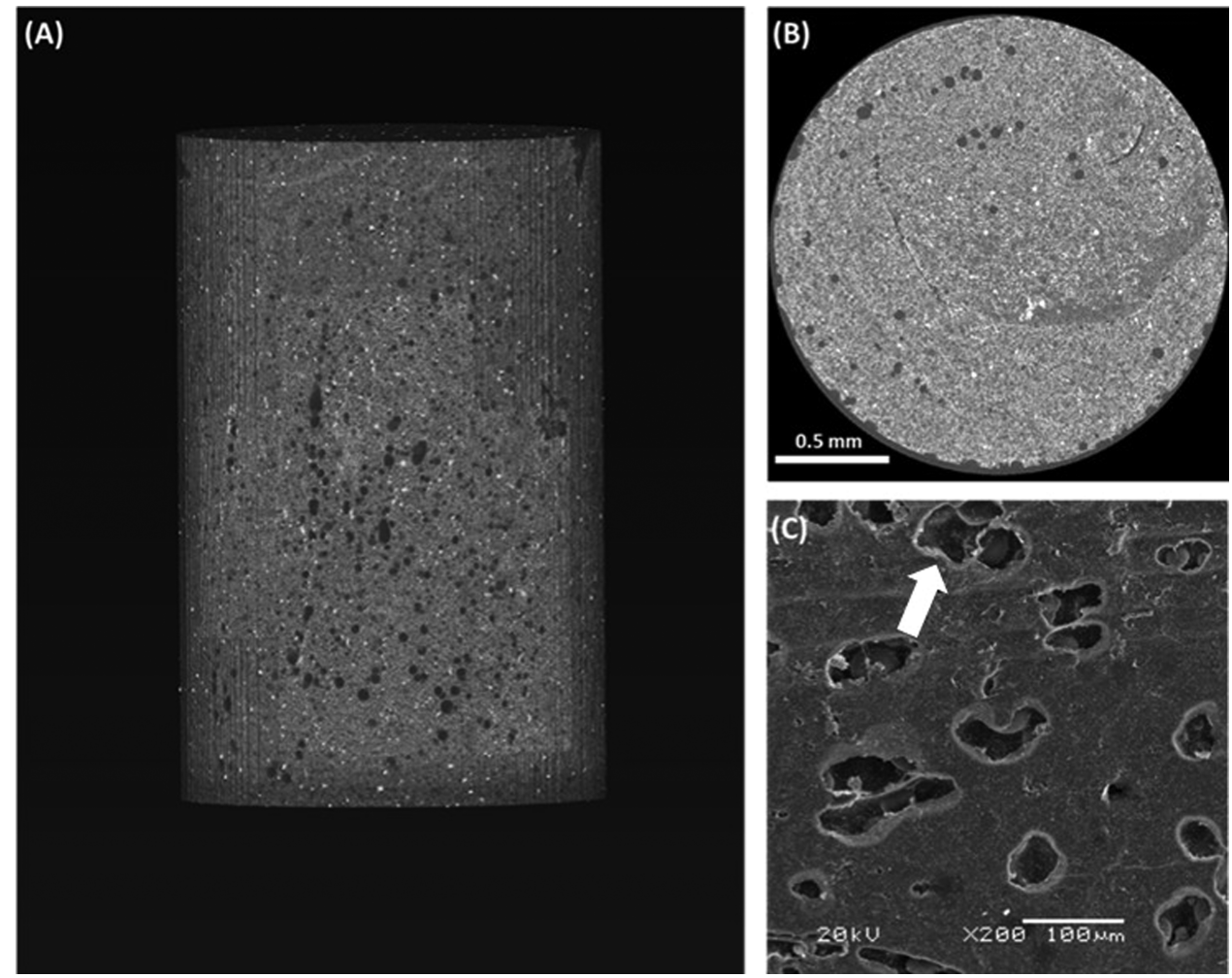

Figure 3. Morphology of PMMA cements. (A) Representative XCT reconstructed volume of a whole PMMA_2:1 cement and (B) cross section of the same specimen. (C) SEM image of PMMA 2:1 cement surface; the arrow shows a PMMA bead within the solidified cement (see Figure S2 for more images).
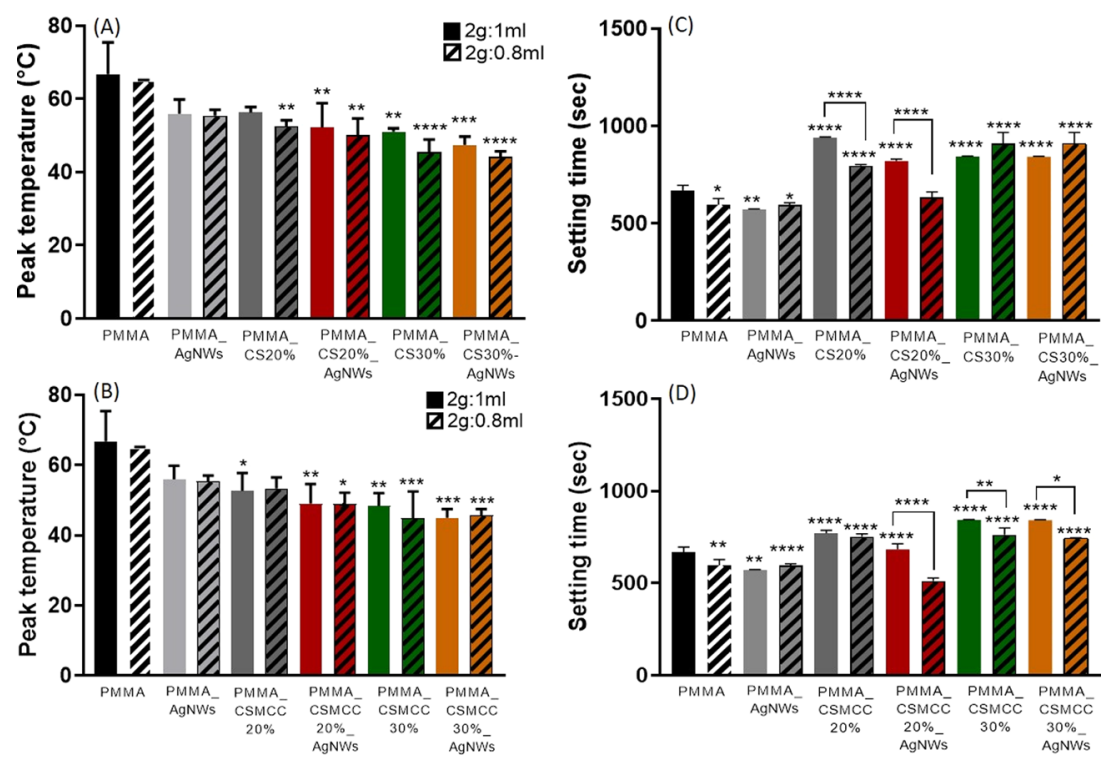

Figure 4. Setting properties of cements. Peak polymerization temperatures of PMMA cements containing CS (A) or CSMCC (B), and setting time of PMMA-based cements, containing CS (C) or CSMCC (D), with different ratios of P/L. Data are reported as a mean \pm SD ( $n=6)$. One-way ANOVA returned $p<0.05$; results of the Dunnett's multicomparison test, used to compare all of the samples with the PMMA control, and results of the $t$-test to compare samples with different $\mathrm{P} / \mathrm{L}$ ratios are reported in the graph $(* p<0.05, * * p<0.01, * * * p<0.001, * * * * p<0.0001)$.

2:0.8, the exothermic reaction temperature was not statistically different $(p>0.05)$. The lowest polymerization temperatures for CS-enriched cements were recorded for PMMA CS30\% _0.8 $\left(45.4 \pm 3.4{ }^{\circ} \mathrm{C}\right)$ and PMMA CS30\%_AgNWs_0.8 (44.2 $\left.\pm 1.4{ }^{\circ} \mathrm{C}\right)$, whereas for CSMCC for PMMA CSMCC $30 \% 0.8$ $\left(44.9 \pm 7.6{ }^{\circ} \mathrm{C}\right)$ and PMMA CSMCC $30 \%$ AgNWs_1 (45.0 \pm $\left.2.5{ }^{\circ} \mathrm{C}\right)$. According to ISO 5833:2002, the ideal setting time for acrylic resin cements, depending on the usage, should be between 3 and $15 \mathrm{~min}$. With increasing concentrations of CS and CSMCC (Figure 4C,D), the setting time increased from $667.2 \pm 28.1 \mathrm{~s}$ (ca. $11 \mathrm{~min}$ ) for PMMA_1 to $843.8 \pm 2.9 \mathrm{~s}$ (ca. 14 min) for PMMA CS30\%_1, remaining within the maximum recommended time. The setting time was lower when the $\mathrm{P} / \mathrm{L}$ ratio was higher. 

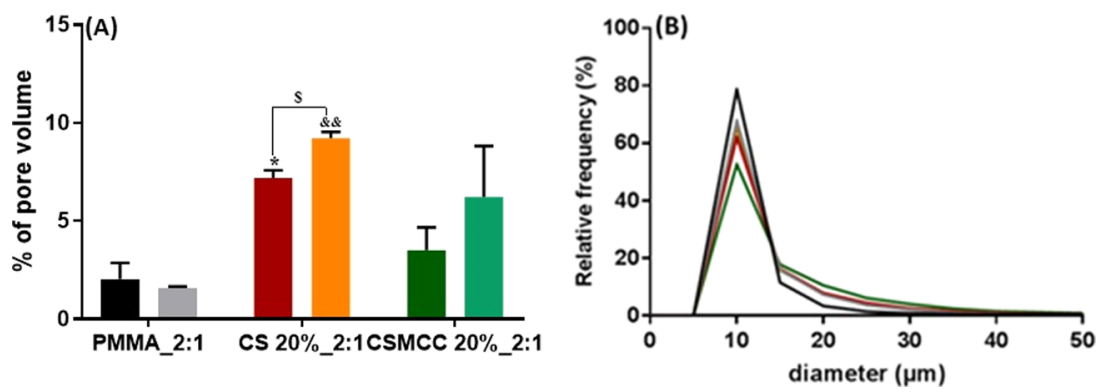

Figure 5. Pore volume and pore size of cements. Percentage pore volume (A) of PMMA (black), PMMA after 4 weeks (gray), CS_20\% (red), CS 20\% after 4 weeks (orange), CSMCC 20\% (dark green), and CSMCC_ $20 \%$ after 4 weeks (light green). Data are reported as a mean \pm SD ( $n$ $=3)$. T-test was performed between the same cement type before and after soaking $\left({ }^{\$} p<0.05\right)$. One-way ANOVA returned $p<0.05(*)$ when comparing the \% pore volume of CS 20\% to PMMA before degradation in PBS; results of the Dunnett's multiple comparison test are reported in the graph. One-way ANOVA returned $p<0.01$ when comparing the \% pore volume of CS_20\% to PMMA after degradation in PBS; results of the Dunnett's multiple comparison test are reported in the graph $(\& \& p<0.01)$. (B) Pore diameter distribution for PMMA (black), PMMA after 4 weeks (gray), CS_20\% (red), CS_20\% after 4 weeks (orange), CSMCC_20\% (dark green), and CSMCC_20\% after 4 weeks (light green).
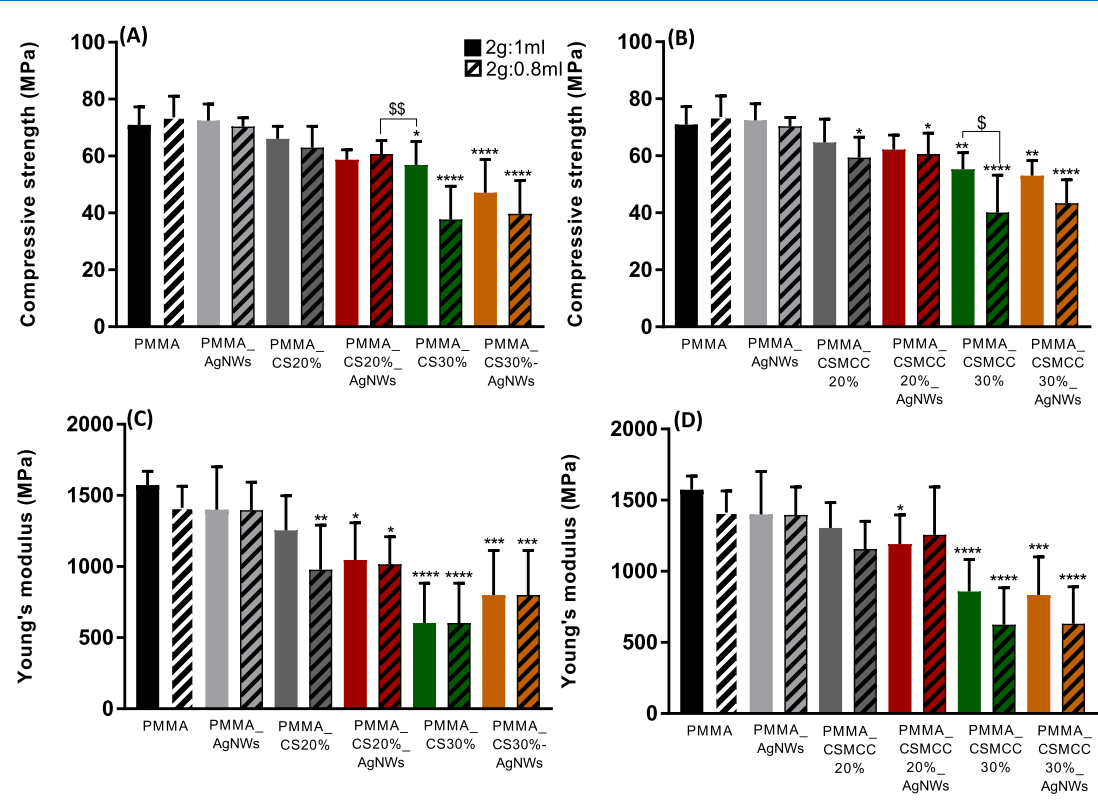

Figure 6. Compressive strength (MPa) and Young's modulus (MPa) of composites cements, containing CS (A, C) and CSMCC (B, D). Results are reported as a mean $\pm \mathrm{SD}(n=6)$. For compressive strength, one-way ANOVA returned $p<0.05$; results of Dunnett's multicomparison test (used to compare all of the samples with the PMMA_2:1) are reported in the graph $(* p<0.05, * * p<0.01, * * * p<0.001, * * * * p<0.0001)$. Unpaired $t$-test was performed to compare each formulation containing a weight-to-volume ratio of $2: 1$ with the respective formulation containing a weight-to-volume ratio of 2:0.8 $\left({ }^{\$} p<0.05,{ }^{\$} p<0.01\right)$. For Young's modulus, one-way ANOVA returned $p<0.05$; results of Dunnett's multicomparison test, used to compare all samples with the PMMA control, are reported in the graph $(* p<0.05)$. Unpaired $t$-test was performed to compare each formulation containing a weight-to-volume ratio of 2:1 with the respective formulation containing a weight-to-volume ratio of $2: 0.8\left({ }^{\$} p<0.05\right)$.

In Vitro Degradation Studies. The porosity and pore size distribution of composite cements were evaluated by XCT. During the polymerization process, voids formed as entrapped air was unable to escape due to the cement paste viscosity. Cements containing biodegradable materials, such as chitosan, were expected to increase in porosity over time, and this was also evaluated by XCT. Samples of the PMMA control and composite cements containing 20\% CS or CSMCC were scanned. The PMMA-CS sample analyzed before degradation showed a significantly higher porosity than PMMA-only $(p<$ 0.05), whereas the PMMA_2:1_CSMCC20\% was not statistically different. Furthermore, the cement containing CS and CSMCC underwent degradation during a 4 week incubation period in PBS $(p<0.05)$, while no significant degradation was shown in the PMMA-only cements (Figure 5A).
An increase in the pore size after 4 weeks of degradation was observed only for PMMA_2:1_CS20\% $(p<0.05)$ with the average pore size increasing from $11.6 \pm 13.4$ to $13.1 \pm 10.9$ $\mu \mathrm{m}$. PMMA cements before and after degradation had a narrower pore size distribution in comparison to the cements containing CS or CSMCC, both before and after degradation (Figure 5B).

To further assess the composite cements, in vitro degradation was evaluated by a gravimetric method. This method is considered the golden standard for in vitro wear assessment. $^{24}$ This provides information about global mass loss, though it does not give any information about the distribution of wear within the material. As shown in Table 1, PMMA-only cements showed a poor weight loss $(0.09 \pm 0.09$ and $0.12 \pm 0.12 \%$ for PMMA_2:1 and PMMA_2:0.8, 
respectively), whereas CS- and CSMCC-based cements presented higher weight loss, which increased with the increase of the polysaccharide concentration, as expected. For instance, the calculated weight loss for PMMA_CS_2:1 samples increased from $0.16 \pm 0.25$ to $0.68 \pm 0 . \overline{57}$ and finally 3.66 $\pm 1.03 \%$ for 10,20 , and $30 \%$ chitosan contents, respectively. No statistical difference between the two MMA concentrations was found. Water absorbance increased with the increase of CS or CSMCC up to nearly $13 \%$ (Table 1 ). Similar trends are reported in the literature for chitosan-based polyester and poly(methyl methacrylate) cements. ${ }^{25}$ This trend can be justified in two different ways: the presence of chitosan that is hygroscopic and promoted the water absorption by the scaffold, while this was not possible in PMMA-only cements that are hydrophobic. Alternatively, higher porosity and/or interconnectivity of the material, due to chitosan degradation, could promote water retention within the scaffold (as suggested from XCT). No statistical difference was determined between CS- and CSMCC-based cements $(p<0.05)$.

Mechanical Properties. In this study, we incorporated increasing mass ratios of CS or CSMCC to study how the mechanical properties of PMMA cements are affected. Surgical PMMA cement is brittle in nature. It is therefore weak under tension but quite strong in compression and is capable of yielding under uniaxial compression. The compressive strength and elastic modulus of composite cements generally decreased, increasing the mass ratio of CS or CSMCC in the mixture (Figure 6). This trend is in good agreement with Dunne et al. for PMMA bone cements (Palacos R) loaded with chitosan, at lower concentrations $(1,3$, and $5 \% \mathrm{w} / \mathrm{w}),{ }^{26}$ and Tan et al. who incorporated $20 \%$ chitosan into PMMA bone cements (CMW endurance bone cement). ${ }^{27}$ When AgNWs were included in the formulation, no statistical differences were reported. This trend has also been reported by Slane et al., who incorporated $1.0 \% \mathrm{w} / \mathrm{w}$ silver nanoparticles into acrylic bone cements. ${ }^{12}$ When the ratio between the powder and liquid was $2: 1$, the compressive modulus and elastic modulus were significantly decreased with the inclusion of $30 \%$ CS. This can be attributed to the presence of less chemical links inside the cements and possibly the presence of loose powder. On the other hand, when the ratio between the powder and liquid was 2:0.8, the compressive strength was significantly different also for lower concentrations of CSMCC (20\%).

The mechanical performance was also evaluated after the in vitro degradation study was carried out for 4 weeks; however, no significant differences were determined with an unpaired $t$ test $(p>0.05)$ (Figures S3 and S4).

Silver Release from Composite Cements. A release study of silver ions was performed in deionized water for a period of 21 days, and the cumulative release profiles are presented in Figure 7. The bone cements loaded with AgNWs continuously released $\mathrm{Ag}^{+}$ions to the surrounding water with the amount of silver increasing significantly within the first day and then reaching a plateau. No statistical difference was found between the different cements studied $(p>0.05)$, even though the cements containing CS/CSMCC presented a higher $\mathrm{Ag}^{+}$ release at any time point.

Antibacterial Properties of Cements. The antimicrobial properties of the composite bone cements were determined using an MTT assay after $24 \mathrm{~h}$ of incubation in an S. aureus suspension. PMMA-only cements presented lower bacterial attachment than the cements containing CS or CSMCC powders at concentrations higher or equal to $20 \%$ (Figure 8 ).

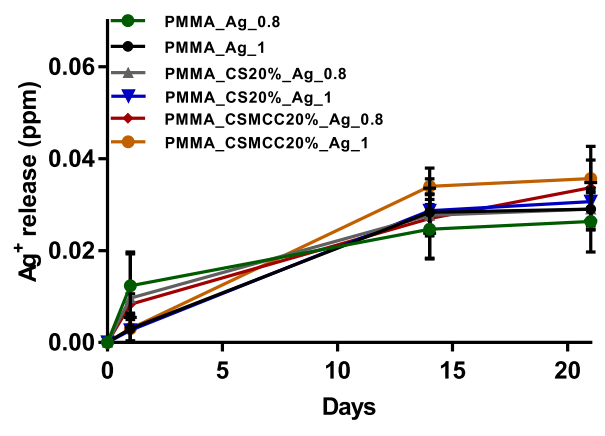

Figure 7. Cumulative release of silver ions from bone cements: PMMA_AgNWs_1 (black), PMMA_AgNWs_0.8 (red), PMMA_CS20\%_AgNWs_1 (blue), PMMA_CS20\%_AgNWs_0.8 (greeñ), PMMA_C $\bar{S} M C C 20 \%$ AgNWs_1 (yellow), PMMA_CSMCC20\%_AgNWs_0.8 (purple). Data are reported as a mean $\pm \mathrm{SD}(n=3)$. One-way ANOVA, at 21 days, returned $p>0.05$.

On the other hand, when AgNWs were incorporated within the formulation, they induced a significant reduction of the viable bacteria attached to the cement for all of the studied formulations $(p<0.05)$, except for CS and CSMCC at $10 \%$.

Evaluation of the Cytocompatibility of the Composite Cements. Cytocompatibility was evaluated both on cement eluates (Figure 9A,B) and directly on composite PMMA cements (Figure 9C,D). The use of eluates simulates the postsurgical release of toxic leachables into the surrounding bony environment. Moreover, through serial dilutions, it is possible to evaluate the dose-dependent effect. Cytocompatibility was assessed after 24 and 48 h of incubation of cells with the eluates, and no statistical difference was found $(p>0.05)$, except for PMMA_AgNWs_1 $(p<0.01)$. The cell viability at $24 \mathrm{~h}$ was $>80 \%$ for all cements with P/L 2:1; except for the cements containing $20 \%$ of CS or CSMCC and AgNWs that caused a significant decrease in cell viability: PMMA_CS20\% _AgNWs_1 (51.6 \pm 31.5\%), PMMA_CSMC $20 \%$ -AgNWs_-1 (55.6 \pm 36.6\%), PMMA_CS20\%_AgNWs_0.8

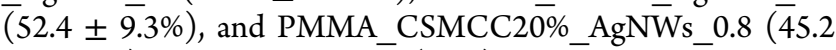
$\pm 30.9 \%)$. Diluted extracts $(50 \%)$ always maintained cell viability $>80 \%$, indicating a dose-dependent effect (Figure S5). When comparing the two different concentrations of MMA at different time points, no statistical difference was found $(p>$ $0.05)$. Overall, these results suggest that with the addition of CS/CSMCC (and consequently the reduction of bone cement powder) in the formulation, less liquid cement could react with the initiator and more toxic leachables were released from the cements over time.

Cell proliferation at 1 and 3 days of seeding cells onto the cement surface was also evaluated. It was found that there was significantly higher cell proliferation between days 1 and 3 for cells seeded on samples containing PMMA-only both with $(p<$ $0.05)$ and without AgNWs $(p<0.0001)$. While for samples containing CS/CSMCC in low amounts, only the following samples showed increased proliferation: PMMA_CS10\% $(p<$ $0.0001)$, PMMA_CS10\%_AgNWs $(p<0.05)$, PMMA_CSMCC10\% $(p<0.05)$, and PMMA_CS20\% $(p<$ $0.05)$. For higher concentrations of polysaccharide, proliferation did not statistically increase $(p>0.05)$. These results are similar to those reported by Tan et al. who loaded PMMAbased cements with chitosan powder at 20\% w/w (PMMAC) ${ }^{28}$ Compared with PMMA, PMMA-C cements presented a lower proliferation rate. These results are also in agreement with the cytotoxicity studies performed on the extracts; the 


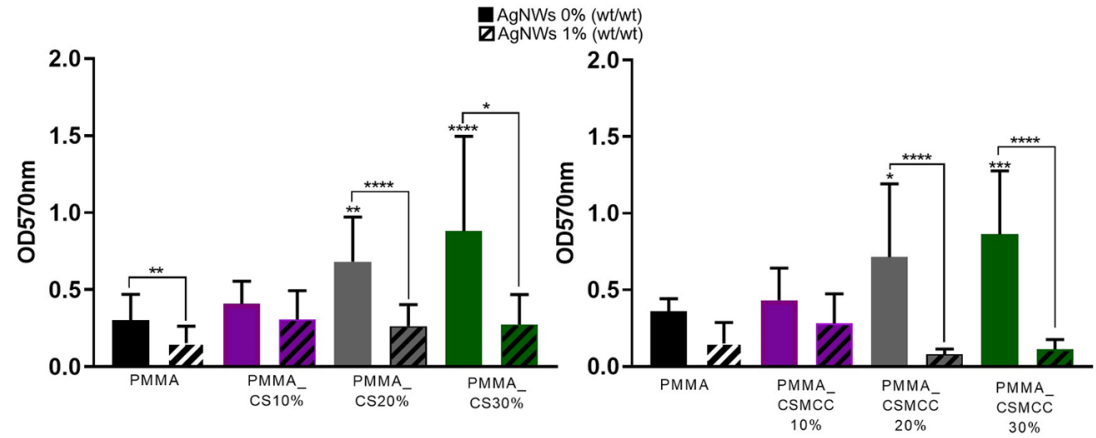

Figure 8. Inhibition of biofilm formation expressed as viable cell count. Absorbance relative to the number of bacterial cells on cement (P/L 2:1) surfaces obtained by an MTT assay after $24 \mathrm{~h}$. (A) PMMA-based cements containing CS. (B) PMMA-based cements containing CSMCC. Data are reported as mean $\pm \mathrm{SD}(n=4)$. One-way ANOVA returned $p<0.05$; results of the post hoc Tukey's multicomparison test are reported in the graph $(* p<0.05)$. Unpaired $t$-test was performed to compare each formulation with and without AgNWs. * represents $p<0.05$, ** represents $p<$ $0.01, * * *$ represents $p<0.001$, and $* * * *$ represents $p<0.0001$.
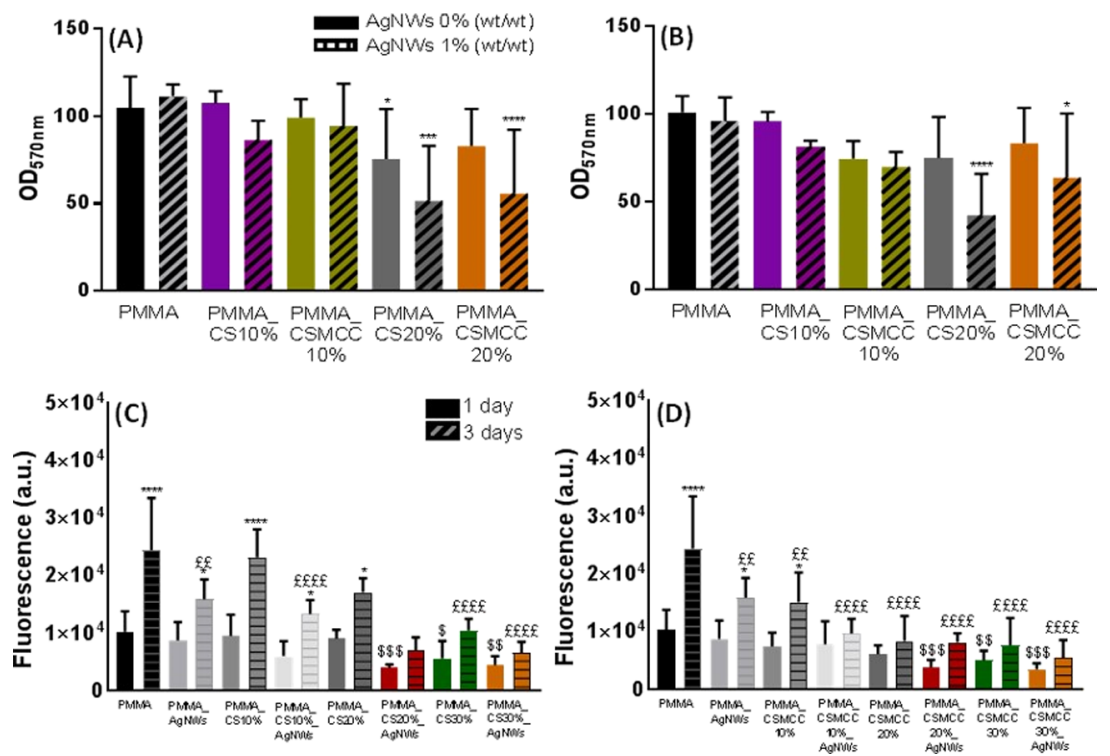

Figure 9. Cytotoxicity of cements on mesenchymal stem cells. Cytotoxicity was tested against the cement eluates (A, B) and by direct contact of cells with cements (C, D). Toxicity of extracts from cements of P/L ratios 2:1 (A) and 2:0.8 (B) at 24 (left column) and $48 \mathrm{~h}$ (right column). Data are reported as mean $\pm \mathrm{SD}(n \geq 3)$. One-way ANOVA returned $p<0.05$; results of the post hoc Tukey's multicomparison test are reported in the graph $(* p<0.05)$. Unpaired t-test was performed to compare each formulation with or without AgNWs. ATP assay for MSCs after 1 day and 3 days of incubation on CS cements (C) and CSMCC cements (D). One-way ANOVA was carried out to compare ATP levels of each cement to PMMA 2:1 at days 1 and 3 and showed a statistical difference $(p<0.05)$. Results of Dunnett's multicomparison are reported in the graph; in particular, ${ }^{\$} p<0.05,{ }^{\$ \$} p<0.01,{ }^{\$ \$} p<0.001$, and ${ }^{\$ \$ \$} p<0.0001$, at day $1 .{ }^{\mathfrak{E}} p<0.05,{ }^{\mathfrak{E E}} p<0.01,{ }^{\mathfrak{E E E}} p<0.001$, and ${ }^{\mathfrak{E E E E}} p<0.0001$, at day 3. The $t$ test was performed to compare each type of cement at 1 and 3 days $(* p<0.05$, **** $p<0.0005)$.

increased ratio between the bone cement liquid and bone cement powder decreased cell viability, over time.

On the other hand, the inclusion of AgNWs did not interfere with cell proliferation in comparison to their controls.

\section{DISCUSSION}

Carefully designing composite biomaterials is paramount in overcoming limitations of currently used products such as PMMA. In the present study, a number of strategies have been combined to address the main limitations affecting the performance of PMMA cements. The strategies included 1 . the addition of biodegradable chitosan to increase material porosity and degradability, thus favoring PMMA/bone integration and tuning the mechanical properties of the material; 2. the use of methacrylated chitosan to reduce polymerization temperature and avoid the risk of tissue necrosis; and 3. the addition of AgNWs to bestow antibacterial properties.

Methacryloyl chitosan (CSMCC) was successfully obtained with a degree of substitution of $25 \%$ and used in the formulation of composite cements. Before addition to the composite cements, both the chitosan and modified chitosan were tested to evaluate their antimicrobial properties in the solid form. The antibacterial activity observed for all chitosan powders studied was lower than that reported in the literature. This was expected, as the powders were in suspension at a neutral $\mathrm{pH}$, while published studies have been carried out at a $\mathrm{pH}$ lower than the $\mathrm{p} K_{\mathrm{a}}$ of chitosan. ${ }^{29}$ In an acidic environment, chitosan is protonated and thus capable of interacting with the negatively charged bacterial surface. ${ }^{16}$ These results confirm the need to add, to composite PMMA cements, other components with antimicrobial properties beside chitosan, to 
obtain an effective action against possible bacterial infections. Once established that CS and CSMCC on their own were not able to provide benefits in terms of the antimicrobial activity, we evaluated their effect on the temperature and time of polymerization. According to ISO 5833:2002, the maximum polymerization temperature for acrylic resin cements should be $\leq 90{ }^{\circ} \mathrm{C}$; temperatures lower than $56{ }^{\circ} \mathrm{C}$ would ensure reduced tissue necrosis at the interface between the cement and the natural tissue. Our study showed that the addition of CS and CSMCC reduced the peak polymerization temperature by heat dissipation throughout the bone cement material as previously shown for other additives, such as $\mathrm{MgO}$, hydroxyapatite, CS, $\mathrm{BaSO}_{4}$, and $\mathrm{SiO}_{2}{ }^{30}$ The addition of the polysaccharide also increased the setting time but within acceptable limits. This experiment revealed that the modification of chitosan to CSMCC did not afford any advantage in terms of significantly reduced polymerization temperature; the simple addition of chitosan was sufficient to achieve this goal. Similarly, the use of CSMCC did not affect water absorption and degradation of the composite cements. However, the use of the polysaccharides allowed us to obtain cements with tunable degradation properties. Similarly, it was expected that chitosan would help modulate the mechanical properties of the composite cements. This would help overcome one of the major problems related to standard PMMA cements that is the mismatch between the mechanical properties of the cement and the bone, especially in the case of osteoporotic bone, ${ }^{31}$ with a consequently increased risk of fracture of the adjacent vertebral bodies, in cases of vertebral augmentation with PMMA. $^{32}$ It has been previously shown that the mechanical properties of PMMA-based bone cements can be decreased by introducing porosity or using additives. ${ }^{26,33}$ The addition of CS and CSMCC resulted in reduced compressive strength and Young's modulus once the concentration of the additive reached $20 \% \mathrm{w} / \mathrm{w}$. This can be due to a combination of increased porosity and incomplete monomer polymerization caused by the presence of the polysaccharide in the setting mixture and in correlation with the observed reduced polymerization temperature. One of the aims of the study was also to create cements that would inhibit the formation of a biofilm in vivo. The lack of antibacterial activity of chitosan powders loaded into PMMA cements was expected as already highlighted by Dunne et al.; this behavior may be due to the physical form of CS or CSMCC powders that act as physical supports for the attachment of bacteria, as previously reported for chitosan microspheres, powders, and flakes. ${ }^{16,34}$ For this reason, AgNWs were added as the antimicrobial agent. We previously demonstrated that AgNWs are able to provide a sustained release of silver ions on their own; ${ }^{14}$ current data support this; in fact, all composite cements released $\mathrm{Ag}^{+}$with a similar profile with the total cumulative release well below the potential toxic limit of $10 \mathrm{ppm}$ for human cells. ${ }^{35}$ Our findings show that AgNWs can reduce biofilm formation on bone cements; these results are in good agreement with those reported by Prokopovich et al. who showed a significant reduction of bacterial attachment on PMMA cements loaded with $0.05 \%$ silver nanospheres $(5.3 \pm 2.3 \mathrm{~nm}) .{ }^{11}$ Chitosan and AgNW combinations in the PMMA cements, similarly to our previous study on composite hydrogels, show that the activity of AgNWs is potentiated by the presence of chitosan due to a synergic activity. ${ }^{14}$ The same combination provides good cell viability when the concentration of chitosan is lower than $20 \%$ $\mathrm{w} / \mathrm{w}$.

\section{CONCLUSIONS}

CS and CSMCC were added to PMMA cements in concentrations ranging from 10 to $30 \% \mathrm{w} / \mathrm{w}$. The reduction of the polymerization temperature was found to be dosedependent and significantly reduced for concentrations $>10 \%$. An increased water uptake and weight loss were observed in cements containing chitosan or modified chitosan in comparison to the controls, and the effect was more noticeable with an increase in their concentration. The compressive strength and elastic modulus generally decreased with an increasing mass ratio of CS or CSMCC in the mixture; the same trend was observed for cytotoxicity. No statistical differences were observed between the materials containing either CS or CSMCC. The results of the in vitro studies demonstrated that the incorporation of AgNWs in PMMAchitosan cements in a concentration of $1 \% \mathrm{w} / \mathrm{w}$ can be a viable approach to prevent $S$. aureus infections on the scaffold, while not affecting their mechanical properties and cytocompatibility. These formulations provided a sustained release of $\mathrm{Ag}^{+}$ions, suggesting potential antimicrobial activity over an extended period of time. Overall, this study suggests that the inclusion of CS/CSMCC (between 10 and 20\%) and AgNWs (1\%) in the existing commercial materials can provide bone cements with good cytocompatibility and appropriate thermal, mechanical, and antibacterial properties.

\section{ASSOCIATED CONTENT}

\section{Supporting Information}

The Supporting Information is available free of charge on the ACS Publications website at DOI: 10.1021/acsomega.9b02290.

Supplementary information (PDF) containing the following figures: Figure S1. Light microscope images of (A) PMMA, (B) CS, and (C) CSMCC particles; Figure S2. SEM images of cement surfaces. $\times 200$ magnification; Figure S3. Compressive strength (A, B) and Young's modulus (C, D) of PMMA-based cements containing $\mathrm{CS}$, before (no border) and after compression (black border). (A, C) Cements with $\mathrm{P} / \mathrm{L} 2: 1$ and (B, D) cements with P/L 2:0.8; Figure S4. Compressive strength (A, B) and Young's modulus (C, D) of PMMAbased cements containing CSMCC, before (no border) and after compression (black border). (A, C) Cements with $\mathrm{P} / \mathrm{L} 2: 1$ and $(\mathrm{B}, \mathrm{D})$ cements with $\mathrm{P} / \mathrm{L} 2$ 2:0.8; Figure S5. Cytotoxicity of cement eluates at different dilutions ( 100 vs $50 \%)$ on mesenchymal stem cells after (A) 1 and (B) 2 days of incubation; Figure S6. Morphology of MSCs cultured on (A) glass slide, (B) PMMA cement, (C) PMMA_AgNWs cement, and (D) PMMA_CS20\% cement, at $\overline{24} \mathrm{~h}$ (PDF)

\section{AUTHOR INFORMATION}

\section{Corresponding Author}

*E-mail: marta.roldo@port.ac.uk.

ORCID

Gianluca Tozzi: 0000-0002-3172-5720

Roger R. Draheim: 0000-0002-7118-1297

Marta Roldo: 0000-0003-2242-7761

Present Address

${ }$ Fitzpatrick Referrals, Surrey, UK (A.S.K.). 


\section{Author Contributions}

The manuscript was written through contributions of all authors. All authors have given approval to the final version of the manuscript.

\section{Funding}

This research has been funded by the Institute of Biological and Biomedical Sciences (IBBS) of the University of Portsmouth.

\section{Notes}

The authors declare no competing financial interest.

\section{ACKNOWLEDGMENTS}

The authors would like to thank Colin Lupton (School of Mechanical Engineering of Portsmouth) for his precious advice on mechanical and thermal studies, and the Zeiss Global Centre (University of Portsmouth) for XCT imaging and image analysis. Moreover, the authors would like to thank Marta Peña Fernández (School of Mechanical Engineering of Portsmouth) for her suggestions on sample preparation for mechanical studies.

\section{ABBREVIATIONS}

AgNWs, silver nanowires; CS, chitosan; MMA, methyl methacrylate; MCC, methacryloyl chloride; CSMCC, methacryloyl chitosan; PMMA, poly(methyl methacrylate)

\section{REFERENCES}

(1) Frazer, R. Q.; Byron, R. T.; Osborne, P. B.; West, K. P. PMMA: An Essential Material in Medicine and Dentistry. J. Long-Term Eff. Med. Implants 2005, 15, 629-639.

(2) Mori, A.; Ohtsuki, C.; Miyazaki, T.; Sugino, A.; Tanihara, M.; Kuramoto, K.; Osaka, A. Synthesis of Bioactive PMMA Bone Cement via Modification with Methacryloxypropyltri-Methoxysilane and Calcium Acetate. J. Mater. Sci. Mater. Med. 2005, 16, 713-718.

(3) Rashid, H.; Sheikh, Z.; Vohra, F. Allergic Effects of the Residual Monomer Used in Denture Base Acrylic Resins. Eur. J. Dent. 2015, 9, 614-619.

(4) Ayre, W. N.; Denyer, S. P.; Evans, S. L. Ageing and Moisture Uptake in Polymethyl Methacrylate (PMMA) Bone Cements. J. Mech. Behav. Biomed. Mater. 2014, 32, 76-88.

(5) Tan, H. L.; Lin, W. T.; Tang, T. T. The Use of AntimicrobialImpregnated PMMA to Manage Periprosthetic Infections: Controversial Issues and the Latest Developments. Int. J. Artif. Organs 2012, 35, 832-839.

(6) Mour, M.; Das, D.; Winkler, T.; Hoenig, E.; Mielke, G.; Morlock, M. M.; Schilling, A. F. Advances in Porous Biomaterials for Dental and Orthopaedic Applications. Materials 2010, 3, 2947-2974.

(7) Khandaker, M.; Meng, Z. The Effect of Nanoparticles and Alternative Monomer on the Exothermic Temperature of PMMA Bone Cement. Procedia Eng. 2015, 105, 946-952.

(8) Olson, M. E.; Horswill, A. R. Staphylococcus Aureus Osteomyelitis: Bad to the Bone. Cell Host Microbe 2013, 13, 629631.

(9) Sanchez, C. J. J.; Ward, C. L.; Romano, D. R.; Hurtgen, B. J.; Hardy, S. K.; Woodbury, R. L.; Trevino, A. V.; Rathbone, C. R.; Wenke, J. C. Staphylococcus Aureus Biofilms Decrease Osteoblast Viability, Inhibits Osteogenic Differentiation, and Increases Bone Resorption in Vitro. BMC Musculoskeletal Disord. 2013, 14, 187.

(10) Ventola, C. L. The Antibiotic Resistance Crisis: Part 1: Causes and Threats. Pharm. Ther. 2015, 40, 277-283.

(11) Prokopovich, P.; Köbrick, M.; Brousseau, E.; Perni, S. Potent Antimicrobial Activity of Bone Cement Encapsulating Silver Nanoparticles Capped with Oleic Acid. J. Biomed. Mater. Res. 2015, 103, 273-281.

(12) Slane, J.; Vivanco, J.; Rose, W.; Ploeg, H.-L.; Squire, M. Mechanical, Material, and Antimicrobial Properties of Acrylic Bone
Cement Impregnated with Silver Nanoparticles. Mater. Sci. Eng., C 2015, 48, 188-196.

(13) Jones, R.; Draheim, R. R.; Roldo, M. Silver Nanowires: Synthesis, Antibacterial Activity and Biomedical Applications. Appl. Sci. 2018, 8, 673 .

(14) De Mori, A.; Hafidh, M.; Mele, N.; Yusuf, R.; Cerri, G.; Gavini, E.; Tozzi, G.; Barbu, E.; Conconi, M.; Draheim, R.; Roldo, M. Sustained Release from Injectable Composite Gels Loaded with Silver Nanowires Designed to Combat Bacterial Resistance in Bone Regeneration Applications. Pharmaceutics 2019, 11, 116.

(15) Stenhagen, I. S. R.; Rukke, H. V.; Dragland, I. S.; Kopperud, H. M. Effect of Methacrylated Chitosan Incorporated in Experimental Composite and Adhesive on Mechanical Properties and Biofilm Formation. Eur. J. Oral Sci. 2019, 127, 81-88.

(16) Ardila, N.; Daigle, F.; Heuzey, M.-C.; Ajji, A. Antibacterial Activity of Neat Chitosan Powder and Flakes. Molecules 2017, 22, 100.

(17) Chang, J.; Huan, Z.; Zhu, H.; Ma, N.; Chen, L.; Zhai, D.; Wu, C. Silicate Bioceramic/PMMA Composite Bone Cement with Distinctive Physicochemical and Bioactive Properties. RSC Adv. 2015, 5, 37314-37322.

(18) Kim, S. B.; Kim, Y. J.; Yoon, T. L.; Park, S. A.; Cho, I. H.; Kim, E. J.; Kim, I. A.; Shin, J.-W. The Characteristics of a HydroxyapatiteChitosan-PMMA Bone Cement. Biomaterials 2004, 25, 5715-5723.

(19) Grela, E.; Kozlowska, J.; Grabowiecka, A. Current Methodology of MTT Assay in Bacteria - A Review. Acta Histochem. 2018, 120, $303-311$.

(20) Zhang, X.; Kang, T.; Liang, P.; Tang, Y.; Quan, C. Biological Activity of an Injectable Biphasic Calcium Phosphate/PMMA Bone Cement for Induced Osteogensis in Rabbit Model. Macromol. Biosci. 2018, 18, No. 1700331.

(21) Dharani, M.; Balasubramanian, S. Synthesis, Characterization and Application of Acryloyl Chitosan Anchored Copolymer towards Algae Flocculation. Carbohydr. Polym. 2016, 152, 459-467.

(22) Endogan, T.; Kiziltay, A.; Kose, G. T.; Comunoglu, N.; Beyzadeoglu, T.; Hasirci, N. Acrylic Bone Cements: Effects of the Poly(Methyl Methacrylate) Powder Size and Chitosan Addition on Their Properties J. Appl. Polym. Sci. 2014, 1313.

(23) Vaishya, R.; Chauhan, M.; Vaish, A. Bone Cement. J. Clin. Orthop. Trauma 2013, 4, 157-163.

(24) Affatato, S.; Zanini, F.; Carmignato, S. Micro X-Ray Computed Tomography Mass Loss Assessment of Different UHMWPE: A Hip Joint Simulator Study on Standard vs. Cross-Linked Polyethylene. PLoS One 2017, 12, No. e0170263.

(25) Abdul Amer, Z. J.; Kareem Ahmed, J.; Fahim Abbas, S. Chitosan/PMMA Bioblend for Drug Release Applications. Int. J. Eng. Technol. 2014, 4, 318-324.

(26) Dunne, N.; Buchanan, F.; Hill, J.; Newe, C.; Tunney, M.; Brady, A.; Walker, G. In Vitro Testing of Chitosan in GentamicinLoaded Bone Cement: No Antimicrobial Effect and Reduced Mechanical Performance. Acta Orthop. 2008, 79, 851-860.

(27) Tan, H.; Ao, H.; Ma, R.; Tang, T. Quaternised ChitosanLoaded Polymethylmethacrylate Bone Cement: Biomechanical and Histological Evaluations. J. Orthop. Transl. 2013, 1, 57-66.

(28) Tan, H.; Guo, S.; Yang, S.; Xu, X.; Tang, T. Physical Characterization and Osteogenic Activity of the Quaternized Chitosan-Loaded PMMA Bone Cement. Acta Biomater. 2012, 8, 2166-2174.

(29) Erdem, B.; Kariptaş, E.; Kaya, T.; Tulumoğlu, Ş.; Görgülü, Ö. Factors Influencing Antibacterial Activity of Chitosan against Aeromonas Hydrophila and Staphylococcus Aureus. Int. Curr. Pharm. J. 2016, 5, 45-48.

(30) Serbetci, K.; Korkusuz, F.; Hasirci, N. Thermal and Mechanical Properties of Hydroxyapatite Impregnated Acrylic Bone Cements. Polym. Test. 2004, 23, 145-155.

(31) Boger, A.; Bisig, A.; Bohner, M.; Heini, P.; Schneider, E. Variation of the Mechanical Properties of PMMA to Suit Osteoporotic Cancellous Bone. J. Biomater. Sci. Polym. Ed. 2008, 19, $1125-1142$ 
(32) Boger, A.; Bohner, M.; Heini, P.; Verrier, S.; Schneider, E. Properties of an Injectable Low Modulus PMMA Bone Cement for Osteoporotic Bone. J. Biomed. Mater. Res. 2008, 86, 474-482.

(33) Knets, I.; Krilova, V.; Cimdins, R.; Berzina, L.; Vitins, V. Stiffness and Strength of Composite Acrylic Bone Cements. J. Achiev. Mater. Manuf. Eng. 2007, 20, 135-138.

(34) Kong, M.; Chen, X. G.; Liu, C. S.; Liu, C. G.; Meng, X. H.; Yu, L. J. Antibacterial Mechanism of Chitosan Microspheres in a Solid Dispersing System against E. coli. Colloids Surf., B 2008, 65, 197-202.

(35) Shivaram, A.; Bose, S.; Bandyopadhyay, A. Mechanical Degradation of $\mathrm{TiO} 2$ Nanotubes with and without Nanoparticulate Silver Coating. J. Mech. Behav. Biomed. Mater. 2016, 59, 508-518. 